\title{
Subcellular lipid droplet distribution in red and white muscles in the obese Zucker rat
}

\author{
J. S. V. Lally • L. A. Snook • X. X. Han • A. Chabowski • \\ A. Bonen • G. P. Holloway
}

Received: 9 September 2011 / Accepted: 10 October 2011 /Published online: 19 November 2011

(C) Springer-Verlag 2011

\begin{abstract}
Aims/hypothesis Little is known about the subcellular distribution of lipids in insulin-resistant skeletal muscle. However, it has recently been suggested that lipid accumulation in the subsarcolemmal region directly contributes to insulin resistance. Therefore we hypothesised that regional differences in lipid distribution in insulin-resistant muscle may be mediated by: (1) a reduction in fatty acid trafficking into mitochondria; and/or (2) a regional increase in the enzymes regulating lipid synthesis.

Methods Transmission electron microscopy was used to quantify lipid droplet and mitochondrial abundance in the subsarcolemmal and intermyofibrillar compartments in red and white muscles from lean and obese Zucker rats. To estimate rates of lipid trafficking into mitochondria, the metabolic fate of radiolabelled palmitate was determined. Key enzymes of triacylglycerol synthesis were also determined in each subcellular region.

Results Subsarcolemmal-compartmentalised lipids represented a small absolute fraction of the overall lipid content in muscle, as regardless of fibre composition (red/white) or phenotype (lean/obese), lipid droplets were more prevalent in the intermyofibrillar region, whereas insulin-resistant white muscles were devoid of subsarcolemmal-compartmentalised lipid droplets. While, in obese animals, lipid droplets accumulated in both subcellular regions, in red muscle of
\end{abstract}

J. S. V. Lally · L. A. Snook - X. X. Han · A. Bonen •

G. P. Holloway $(\bowtie)$

Department of Human Health and Nutritional Science,

University of Guelph,

Guelph, ON, Canada N1G 2W1

e-mail: ghollowa@uoguelph.ca

A. Chabowski

Department of Physiology, Medical University of Bialystok,

Bialystok, Poland these animals lipids only appeared to be trafficked away from intermyofibrillar mitochondria, a process that cannot be explained by regional differences in the abundance of triacylglycerol esterification enzymes.

Conclusions/interpretation Lipid accumulation in the subsarcolemmal region is not necessary for insulin resistance. In the intermyofibrillar compartment, the diversion of lipids away from mitochondria in insulin-resistant animals probably contributes to lipid accumulation in this subcellular area.

Keywords Lipid droplets $\cdot$ Mitochondria $\cdot$ Subcellular lipids · Transmission electron microscopy · Triacylglycerol
Abbreviations
DGAT1 Diacylglycerol acyltransferase 1
GPAT1 Glycerol-3-phosphate acyltransferase 1
PPAR Peroxisome proliferator-activated receptor
TEM Transmission electron microscopy

\section{Introduction}

Insulin resistance is associated with increased circulating lipids, as well as ectopic lipid accumulation in skeletal muscle $[1,2]$. In this tissue, intramuscular lipid accumulation seems to occur in two distinct intracellular compartments, namely the subsarcolemmal region located directly beneath the sarcolemmal membrane and in an intermyofibrillar region between the myofibrils [3]. These compartments also harbour subsarcolemmal and intermyofibrillar mitochondria, and therefore intramuscular lipid depots are found in close proximity to mitochondria [4]. Recently it has been suggested that the subcellular distribution of intramuscular 
lipid droplets in the subsarcolemmal region, rather than the total content of various lipid species, contributes to attenuation of insulin signalling in muscle [5]. There are several potential mechanisms that could result in subcellular variations in intramuscular lipid accretion, including regionally selective impairments of mitochondrial fatty acid oxidation and/or increased abundance of the enzymes involved in triacylglycerol esterification.

Subsarcolemmal and intermyofibrillar mitochondria differ in several characteristics, including size, enzymatic activities and rates of fatty acid oxidation [6-10]. In addition, rates of substrate utilisation in these mitochondrial subpopulations respond differently to various physiological perturbations, including aerobic training-induced adaptations [11], mitochondrial biogenesis induced by peroxisome proliferator-activated receptor (PPAR) co-activator $1 \alpha$ $(\mathrm{PGC}-1 \alpha)$ [12], pharmacological activation of PPAR [13] and obesity [9]. In Zucker diabetic fatty rats, a model of severe type 2 diabetes, subsarcolemmal, but not intermyofibrillar mitochondrial number, width and density were increased by $50 \%$ to $70 \%$, although fatty acid oxidation rates were increased to similar degrees in subsarcolemmal and intermyofibrillar mitochondria [14]. Thus, differences in subsarcolemmal and intermyofibrillar mitochondrial morphology and fatty acid metabolism may be associated with differences in lipid accumulation in proximity to subsarcolemmal and intermyofibrillar mitochondria, particularly in insulin-resistant muscle in which fatty acid transport into muscle is known to be upregulated $[9,15]$.

On the other hand, lipid accumulation in specific subcellular compartments in muscle may be associated with regional increases in enzymes regulating the rate of triacylglycerol synthesis. Intramuscular lipid droplets are visible via transmission electron microscopy (TEM) and are thought to largely reflect triacylglycerol content $[14,16]$. Triacylglycerol esterification can be regulated by a number of enzymes, which in most cell types reside on the endoplasmic reticulum, with the exception of glycerol-3phosphate acyltransferase 1 (GPAT1), which is located on mitochondria [17]. This raises the possibility that partitioning of fatty acyl-CoA to storage or oxidation can occur in close proximity to the mitochondria, a possibility also implied by the intimate spatial relationship between mitochondria and lipid droplets $[5,14,18,19]$. However, it is currently unknown whether: (1) the enzymes involved in triacylglycerol synthesis are present and differentially associated in subsarcolemmal and/or intermyofibrillar mitochondrial compartments in skeletal muscle; and (2) whether the subcellular presence of these enzymes is altered in muscles from insulin-resistant animals.

Together, this amounts to tentative evidence that differences in subsarcolemmal and intermyofibrillar mitochondrial fatty acid oxidation and differences in subsarcolemmal and intermyofibrillar lipid accumulation are associated. However, in general, little is known about: (1) the possibility of divergent lipid accumulation patterns in subcellular regions in either highly oxidative (red) and glycolytic (white) muscles with inherently different capacities for lipid metabolism; (2) whether these patterns of lipid accretion are altered in obesity; and (3) whether different lipid accumulation patterns in skeletal muscle are associated with insulin resistance in this tissue. Therefore, in the present study, we examined the accumulation of intramuscular lipids in proximity to subsarcolemmal and intermyofibrillar mitochondrial regions in red and white skeletal muscle of lean and obese Zucker rats, a well-known animal model of obesity and insulin resistance. We also examined, in intact muscle, the levels of radiolabelled palmitate accumulation in subsarcolemmal and intermyofibrillar mitochondria. Our results show that in obese animals excess lipids accumulate primarily in the intermyofibrillar regions of skeletal muscle, as lipid droplet size and density are greatly increased. At the same time, incoming fatty acids are trafficked away from intermyofibrillar mitochondria.

\section{Methods}

\section{Animals}

Female lean $(246 \pm 8 \mathrm{~g})$ and obese $(400 \pm 8 \mathrm{~g})$ Zucker rats aged 10 weeks (Charles River, Baie d'Urfé, QC, Canada) were housed in a climate control facility at the University of Guelph, and allowed free access to rat chow and water. All experiments were performed using procedures approved by the University of Guelph Animal Care Committee.

\section{Basal and insulin-stimulated glucose uptake}

Basal and insulin-stimulated 3-O-methylglucose uptake was measured using a submaximal concentration of insulin $(150 \mu \mathrm{U} / \mathrm{ml})$ in perfused rat hindlimb muscles as we have described previously $[12,20]$.

Biochemical determination of intramuscular lipid content

Intramuscular levels of triacylglycerol, diacylglycerol and ceramide were measured as described previously [21].

Transmission electron microscopy

Sample preparation, sectioning and TEM imaging were performed as we have described previously [14, 16]. Following tissue preparation, samples were embedded in resin (London Resin Company, London, UK) and $100 \mathrm{~nm}$ longitudinal sections were cut, mounted on 200 mesh formvar/carbon copper grids (CanEMco, Lakefield, PQ, 
Canada), and stained with $2 \%$ uranyl acetate (wt/vol.) and Reynold's lead citrate. A minimum of three sections was laid on to each grid (range 3-5) and labelled with serial numbering such that individuals quantifying images were blinded to the phenotype of the animals. Images were obtained from each animal in a randomised systematic order from multiple fibres, including several images acquired at a magnification of $\times 46,000, \times 10,500, \times 5,800$ or $\times 3,400$ using a CM 10 TEM (Phillips, Hillsboro, OR, USA) at $80 \mathrm{kV}$ and a digital charge-coupled device (CCD) camera (Olympus/SIS Morada, Richmond Hill, ON, Canada).

\section{Quantification of TEM images}

We generated 477 images, which were quantified by three investigators. Inter- and intra-investigator variability tests showed no signs of bias and low coefficients of variation $(<10 \%)$. All images were analysed for mitochondrial variables as we have described previously $[14,16]$. We analysed lipid droplet density in two ways, counting (1) the number of lipid droplets in subsarcolemmal and intermyofibrillar regions normalised to muscle area and (2) the relative percentage of subsarcolemmal lipids. The latter was calculated by determining subsarcolemmal lipids normalised to muscle fibre surface area and intermyofibrillar lipids normalised to the volume beneath the surface area, assuming an average radius of $40 \mu \mathrm{m}$ and cylindrical shape of the fibres as reported by others [5]. The cross-sectional area of individual lipid droplets was determined using a software package (iTEM; Olympus/SIS).

\section{Mitochondrial DNA}

Mitochondrial DNA content was determined in the red and white portions of the tibialis anterior muscle using real-time PCR as we have previously reported $[12,14]$.

Whole-muscle palmitate metabolism

Rates of palmitate esterification and oxidation were determined in soleus muscles as previously described [22].

Palmitate trafficking to mitochondria

Soleus muscle strips were prepared as outlined previously [22]. Muscles were incubated for $60 \mathrm{~min}$ in the presence of $37 \mathrm{kBq}\left[{ }^{3} \mathrm{H}\right]$ palmitate (GE Healthcare, Baie D'Urfe, PQ, Canada) and $150 \mu \mathrm{U} / \mathrm{ml}$ insulin. Thereafter, mitochondria were isolated as previously published [23-25], protein contents were determined using a BCA assay and $100 \mu \mathrm{l}$ of the radiolabelled mitochondrial suspension was counted using scintillation counting. Counts were normalised to protein content. Preliminary time course experiments showed that lipid accretion was linear for the duration of the $60 \mathrm{~min}$ incubation.

\section{Western blotting}

Western blotting was performed as previously described [23-25]. Antibodies used were: GPAT1 (Abcam, Cambridge, MA, USA), diacylglycerol acyltransferase (DGAT1) (Abcam), lipin 1 (Abcam), adipose triacylglycerol lipase (Cayman Chemical, Ann Arbor, MI, USA) and hormonesensitive lipase (Cell Signaling Technology, Danvers, MA, USA).

\section{Statistical analysis}

Data were analysed using two-tailed unpaired $t$ tests or 2-way ANOVA, and when appropriate Fisher's least significant difference post hoc tests. A value of $p<0.05$ was considered significant. All data are reported as mean \pm SEM.

\section{Results}

Skeletal muscle insulin resistance

Independent of phenotype, basal and insulin-stimulated rates of glucose transport were greater in red than in white muscles (Fig. 1a, b). However, in obese animals, the basal rates of glucose transport were reduced by $\sim 30 \%$ in both muscle types (Fig. 1a). In addition, insulin sensitivity was reduced by $\sim 75 \%$ in red and white muscles of the obese animals (Fig. 1b).

\section{Intramuscular lipids}

Intramuscular lipid content was determined in two ways. Traditional biochemical analyses were used to quantify intramuscular triacylglycerol, diacylglycerol and ceramide content. In addition, we used TEM images to determine lipid droplet accumulation in selected subcellular compartments.

Biochemical determinations Regardless of muscle fibre composition, triacylglycerol (red $\sim 3.7$-fold, white $\sim 4.2$ fold) and diacylglycerol (red 37\%, white 85\%) contents were increased in obese animals (Fig. 2a, b). Ceramide content was increased in red (39\%), but not in white muscles of the obese animals (Fig. 2c).

Lipid droplet size, number and subcellular distribution

TEM images clearly showed that visible intramuscular lipid droplets: (1) were distributed in specific subcellular muscle 

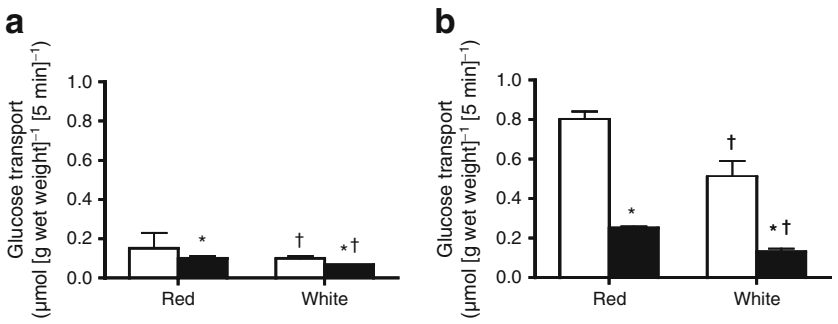

Fig. 1 Basal (a) and insulin-stimulated (b) glucose uptake in red and white muscles by wet weight (g) from lean (white bars) and obese (black bars) Zucker rats. Bars are mean \pm SEM, based on $n=5$ independent experiments; ${ }^{*} p<0.05$ for difference from lean animals; ${ }^{\dagger} p<0.05$ for difference from red

compartments in proximity to subsarcolemmal and intermyofibrillar mitochondria; (2) differed substantially in lean and obese animals; and (3) displayed the expected muscle distribution (i.e. greater in red than white) (Fig. 3 a-f). Lipid droplets in lean and obese white muscle were not prominent (Fig. 3f). Thus, the comparisons between red and white muscles below are necessarily based on the relatively few regions of white muscle where lipid droplets were observed.

Individual lipid droplet size and number In all muscles examined, regardless of muscle and phenotype, lipid droplets were more prevalent (approximately four- to fivefold) in the intermyofibrillar than in the subsarcolemmal region (Fig. 3a-f). In lean and obese animals there were also considerable differences in lipid droplets between red and white muscles, as well as between respective muscles within these animals (Fig. 3a-f).

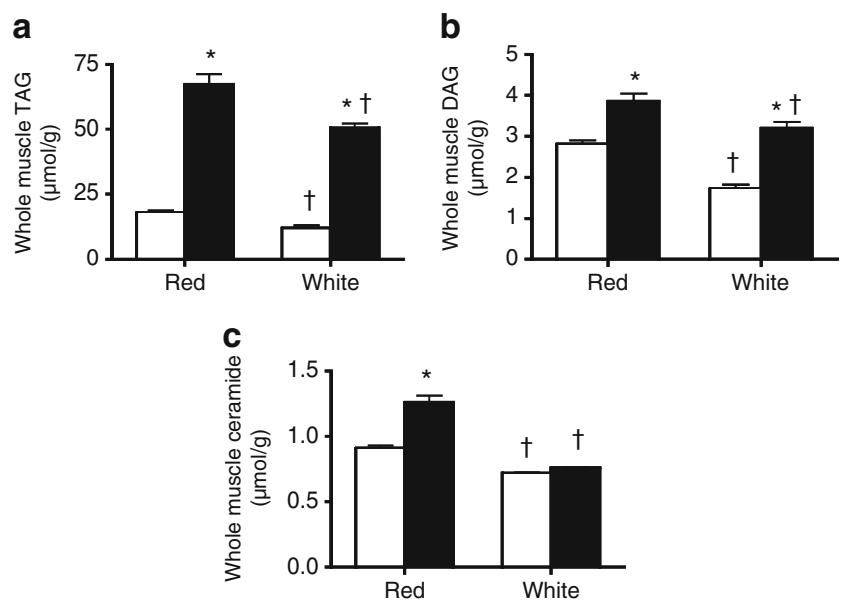

Fig. 2 Biochemical determination of intramuscular lipid species in red and white muscles from lean (white bars) and obese (black bars) Zucker rats. a Triacylglycerol (TAG), (b) diacylglycerol (DAG) and (c) ceramide contents. Bars represent mean \pm SEM, based on $n=5$ independent experiments; ${ }^{*} p<0.05$ for difference from lean animals; ${ }^{\dagger} p<0.05$ for difference from red a

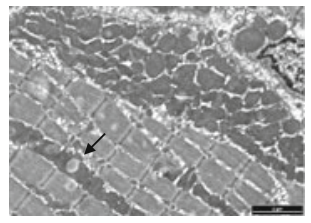

C

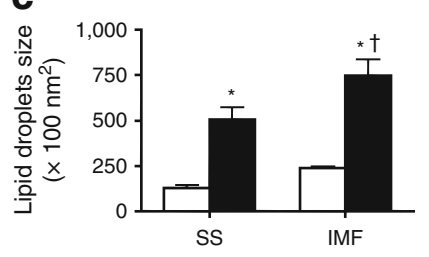

e

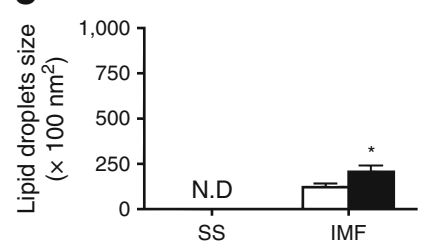

b

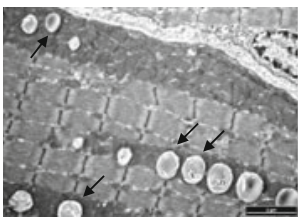

d

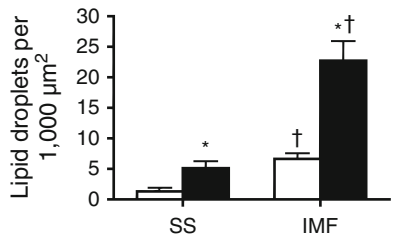

f

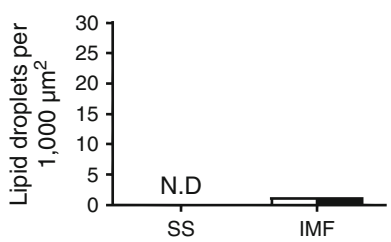

Fig. 3 Subcellular lipid deposition in red and white muscles from lean and obese Zucker rats. Representative TEM images demonstrating the differences in lipid droplet abundance in the subsarcolemmal (SS) and intermyofibrillar (IMF) regions in red muscle from lean (a) and (b) obese Zucker rats. Scale bar $2 \mu \mathrm{m}$, magnification $\times 10,500$. Similar images were used to quantify lipid droplet number in red (d) and white (f), and droplet size in red (c) and white (e) muscles, respectively, from lean (white bars) and obese (black bars) animals. Bars represent the mean \pm SEM, based on $n=5$ independent experiments; ${ }^{*} p<0.05$ for difference from lean animals; ${ }^{\dagger} p<0.05$ for difference from subsarcolemmal

Relative to red muscles in lean animals, the red muscle of obese animals contained considerably more individual lipid droplets per unit area of muscle, both in the subsarcolemmal (approximately fourfold) and the intermyofibrillar (approximately threefold) mitochondrial regions (Fig. 3d). Moreover, whereas the size of individual lipid droplets was increased approximately threefold in the subsarcolemmal and intermyofibrillar regions of obese animals (Fig. 3c), the number of lipid droplets in the latter region was approximately fivefold greater than in the subsarcolemmal region (Fig. 3d). Thus when normalised to fibre surface area, subsarcolemmal lipids only represented a small fraction of the overall lipids in red muscle (lean $5.4 \pm 0.5 \%$, obese $9.6 \pm 0.5 \%$ ).

In white muscle, in contrast, lipid droplets were barely present in the subsarcolemmal compartments of lean or obese animals (Fig. 3e, f). The numbers of lipid droplets in the intermyofibrillar region were comparable in lean and obese animals (Fig. 3f). In white muscle, therefore, the increase in total triacylglycerol content (Fig. 2b) appears to be due exclusively to increased droplet size in the limited 
number of lipid droplets in the intermyofibrillar region (Fig. 3e).

\section{Mitochondrial size and density}

Since altered mitochondrial structure and lipid metabolism have been implicated in the development of insulin resistance, we next examined the size, number and density of subsarcolemmal and intermyofibrillar mitochondria in longitudinal sections of red and white muscles from lean and obese animals (Fig. 4a-d).

Mitochondrial size In general, the qualitative appearance of mitochondria in lean and obese animals did not differ with respect to cristae density. In red muscle, there was no difference in size of individual subsarcolemmal mitochondria in lean and obese animals. In contrast, intermyofibrillar mitochondria were larger (by 58\%) in the obese animals (Fig. 4a).

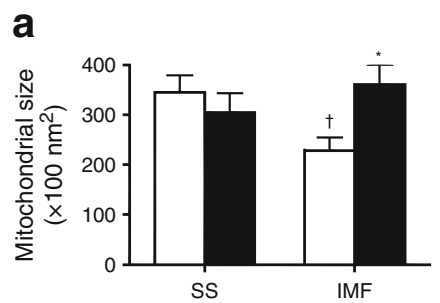

C

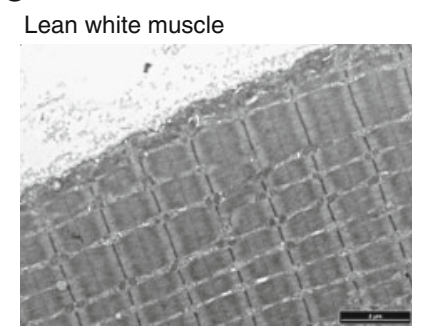

Obese white muscle

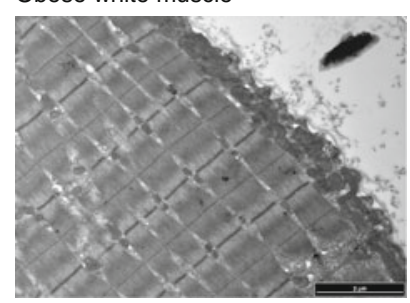

In white muscle, mitochondria were smaller (by $\sim 50 \%$ ) and less prevalent (Fig. 4c) than in red muscle, regardless of phenotype. In white muscle, moreover, subsarcolemmal mitochondria were rarely observed, while intermyofibrillar mitochondria appeared highly organised in doublets (Fig. 4c) flanking the Z-lines, as has been seen by others [26]. Despite the scarcity of white muscle subsarcolemmal and intermyofibrillar mitochondria, these, when detected, did not differ in size in the two subcellular regions of lean or obese animals (Fig. 4b).

Mitochondrial density in red muscle In obese animals, red muscle mitochondrial density was increased in the subsarcolemmal (by 21\%) and intermyofibrillar (by 27\%) mitochondrial regions (Fig. 4d), suggesting an increase in mitochondrial content. This is supported by an increase in red muscle mDNA (Fig. 4e). Given the paucity of subsarcolemmal and intermyofibrillar mitochondria and their diffuse nature in white muscle, we did not quantify mitochon-

b

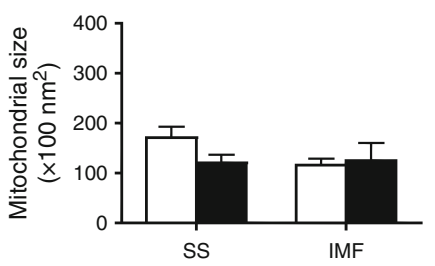

d

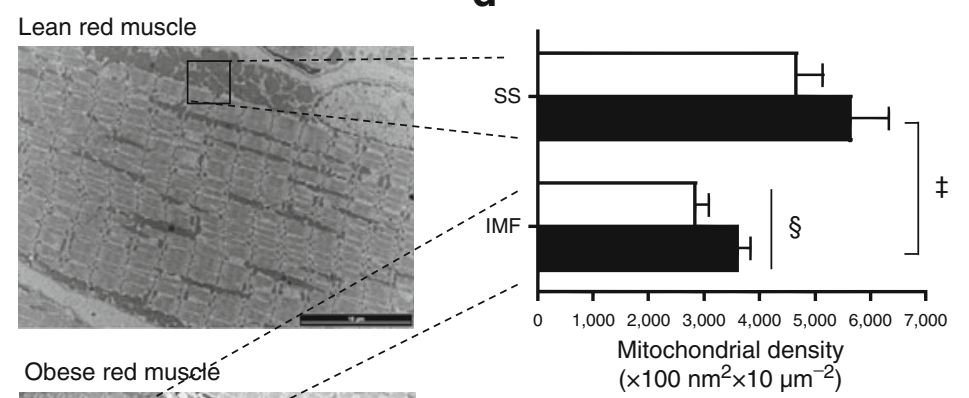

e

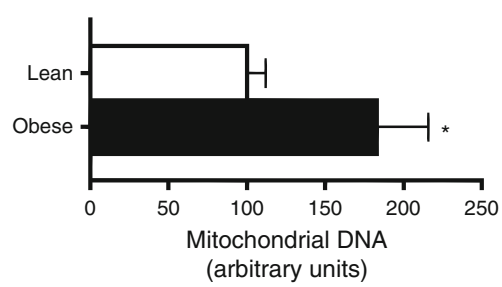

Fig. 4 Mitochondrial subcellular characteristics in red and white muscles from lean (open bars) and obese (closed bars) Zucker rats. Subsarcolemmal (SS) and intermyofibrillar (IMF) mitochondrial size in red (a) and white (b) muscle was determined in TEM images (c). Mitochondrial density in both SS and IMF regions (d) and mitochondrial DNA (e) are presented for red muscle only, as the diffuse nature of mitochondria within white muscle prevented TEM analysis. Bars $(\mathbf{a}, \mathbf{b}, \mathbf{d}, \mathbf{e})$ represent the means \pm SEM based on $n=5$ independent experiments. The scale bar represents $2 \mu \mathrm{m}$ for white muscle and $10 \mu \mathrm{m}$ for red muscle. Representative images were taken at $\times 10,500$ (obese white muscle), $\times 7,900$ (lean white muscle) and $\times 3,400$ (lean and obese red muscle) magnification. *Significantly different from lean animals $(p<0.05)$; $\uparrow$ significantly different from subsarcolemmal $(p<0.05)$; $\ddagger$ for main effect of phenotype (lean vs obese) $(p<0.05)$; and $\S$ for main effect of subcellular location (subsarcolemmal vs intermyofibrillar) $(p<0.05)$ 
drial density in the two regions of this tissue. However, whole muscle mDNA was increased by $26 \%$ in white muscle of obese animals (lean $63 \pm 3$ vs obese $79 \pm 8$ arbitrary units), suggesting that, similarly to red muscle, mitochondrial content was increased in this animal group.

Metabolic trafficking of palmitate

As lipid droplets were found, without exception, to be in direct contact with mitochondria (Fig. 5a), we next
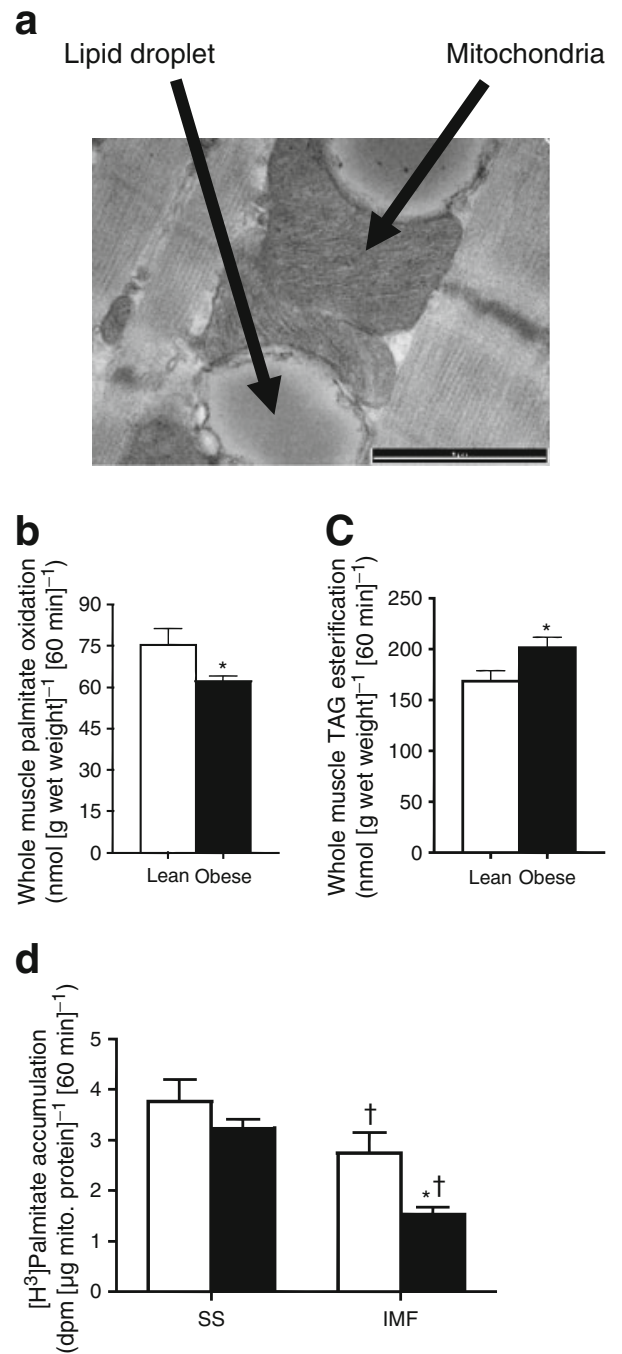

Fig. 5 Intramuscular metabolic fate of palmitate in red muscles from lean and obese Zucker rats. a Representative image demonstrating the intimate association between lipid droplets and mitochondria. Scale bar $1 \mu \mathrm{m}$, magnification of representative image $\times 46,000$. b Palmitate oxidation and triacylglycerol (TAG) esterification (c) in incubated soleus muscle by wet weight (g). White bars, lean animals; black bars, obese. d Accumulation of $\left[{ }^{3} \mathrm{H}\right]$ palmitate in the subsarcolemmal (SS) and intermyofibrillar (IMF) mitochondria isolated from incubated soleus muscles; mito., mitochondrial. Bars represent the mean \pm SEM from $n=6$ independent experiments; ${ }^{*} p<0.05$ for difference from lean animals; ${ }^{\dagger} p<0.05$ for difference from subsarcolemmal examined the metabolic fate of palmitate in order to determine the potential cause of lipid accumulation in the obese animals. Palmitate trafficking experiments were not performed in white muscle, as lipid droplets were only prevalent in the red muscle.

Palmitate oxidation and triacylglycerol esterification In the obese animals, whole-muscle palmitate oxidation was reduced by $\sim 20 \%$ (Fig. 5 b), while rates of triacylglycerol esterification were increased (by $20 \%$ ) (Fig. 5c). Combined, these data suggest that fatty acids are trafficked into lipid droplets.

To further examine this, we determined fatty acid accumulation in the subsarcolemmal and the intermyofibrillar mitochondrial compartments following $\left[{ }^{3} \mathrm{H}\right]$ palmitate exposure $(60 \mathrm{~min})$ in an incubated soleus muscle preparation. In obese animals, the accumulation of radiolabelled fatty acids in subsarcolemmal mitochondria was unaltered (Fig. 5d). In contrast, fatty acid accumulation in intermyofibrillar mitochondria was reduced by $44 \%$ (Fig. 5d).

Triacylglycerol esterification enzyme content and subcellular distribution Given the apparent trafficking of lipids away from intermyofibrillar mitochondria, we next examined whether the enzymes involved in fatty acid esterification were present and increased specifically in intermyofibrillar mitochondria in obese animals, thereby directing fatty acids towards triacylglycerol synthesis and away from mitochondrial oxidation. However, despite the functional differences in the metabolic fate of palmitate in obese animals, there were no differences in the total cellular content of selected enzymes involved in triacylglycerol metabolism (GPAT1, lipin 1 and DGAT1) (Fig. 6a-c). In addition, while GPAT1 was detected in isolated subsarcolemmal and intermyofibrillar mitochondria, GPAT1 content was not altered with obesity in either region. In contrast, lipin 1 and DGAT1 were not detected in isolated mitochondria (Fig. 6b, c).

\section{Discussion}

In the current study, we examined the subcellular distribution of lipids in red and white muscles of lean and insulinresistant animals. Our data demonstrate that: (1) lipid droplets are more prevalent in the intermyofibrillar region in lean and obese animals; (2) insulin-resistant white muscles are devoid of subsarcolemmal lipid droplets and therefore (3) subsarcolemmal lipids are not required for insulin resistance; and (4) within the intermyofibrillar region, lipids are trafficked away from mitochondria in obese animals, a process that (5) cannot be explained by 


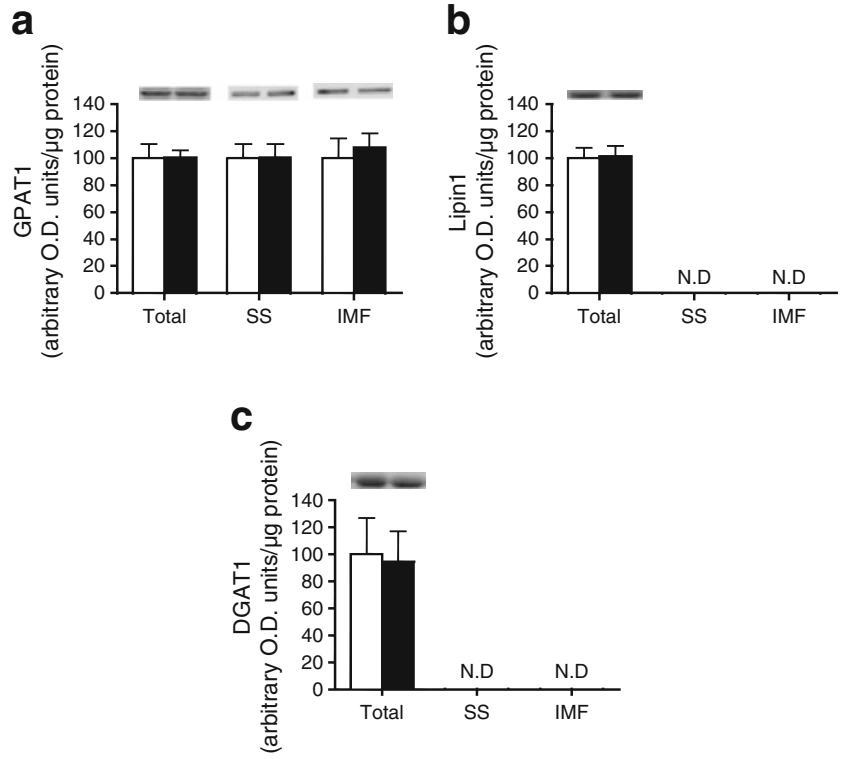

Fig. 6 Protein content and subcellular distribution of triacylglycerol esterification enzymes in red and white muscles from lean (white bars) and obese (black bars) Zucker rats. a GPAT1, (b) lipin 1 and (c) DGAT1 were measured at the whole-muscle level, as well as in subsarcolemmal (SS) and intermyofibrillar (IMF) mitochondria. Bars represent the mean \pm SEM in $n=5$ independent experiments; ${ }^{*} p<0.05$ for difference from lean animals. N.D., not detected with antibody; O.D., optical density

increased abundance of triacylglycerol esterification enzymes. This diversion of lipids away from intermyofibrillar mitochondria probably contributes to lipid accumulation in this subcellular compartment in insulin-resistant muscle.

Total cellular lipids and insulin resistance

Skeletal muscle is key for glucose disposal and is known to take up $\sim 80 \%$ of a glucose load [27]. An increase in the intramuscular content of selected lipids has been associated with skeletal muscle insulin resistance [28-30]. Our findings here support this notion, as total intramuscular triacylglycerol and diacylglycerol contents were increased in the red and white muscles examined in the obese animals. In addition, red, but not white, skeletal muscle from obese animals displayed increased ceramide content. It is currently believed that the accumulation of bioactive lipids, such as diacylglycerol [31] and ceramides [32], contributes to the development of insulin resistance $[31,32]$. In the current study, the white muscle of obese animals was insulin-resistant, which coincided with increased diacylglycerol, but unaltered ceramide content. Combined, these data suggest that increased ceramide content is not necessarily a pre-requisite for insulin resistance, supporting instead the belief that diacylglycerol content may have a direct role in this condition.
While it was originally thought that the accumulation of triacylglycerol was a direct cause of insulin resistance [30], the view now is that triacylglycerol is a neutral and beneficial lipid storage depot, and that the accumulation of triacylglycerol in insulin resistance skeletal muscle is likely to be a marker of altered lipid homeostasis, rather than a cause of insulin resistance. This view is supported by a number of observations, including the observation that overabundance of DGAT1 increases intramuscular triacylglycerol content, while protecting against diet-induced insulin resistance [33], as well as the finding that exercise-trained athletes display high intramuscular triacylglycerol content, but are highly insulin-sensitive [34, 35]. However, a recent report by Nielsen and colleagues [5] suggests that triacylglycerol lipid droplets can negatively affect insulin signalling if accumulated in the subsarcolemmal subcellular region, thus renewing interest in triacylglycerol accumulation as a potential cause of insulin resistance.

\section{Subcellular distribution of lipids}

Regardless of fibre composition or genotype, we found that lipid droplets were larger and more prevalent in the intermyofibrillar region. Moreover, our calculation of the relative abundance of subsarcolemmal lipids, based on assumptions of average fibre diameter and shape in red muscle, suggested that subsarcolemmal lipids only represented approximately $6 \%$ to $10 \%$ of the overall lipids in the muscle. Lipid droplet content in the intermyofibrillar region increased to a greater absolute magnitude in the muscle fibre of obese Zucker rats. However, given that subsarcolemmal lipids are virtually absent in lean animals, subsarcolemmal lipids increased to a greater relative amount with obesity. Combined, these data imply that lipid droplet accumulation in various subcellular regions in obese animals reflects alterations in total cellular lipid homeostasis. While it is possible that estimates based on fibre diameter and shape underestimated the relative content of subsarcolemmal lipids in the cell, lipid droplets were not detectable in the subsarcolemmal region of white muscle even when this was insulin-resistant, suggesting that lipid droplets in the subsarcolemmal region are not required for the development of insulin resistance. This is in contrast to a recent study by Nielsen et al. [5], who found that increased lipid droplet volume was exclusively limited to the subsarcolemmal compartment in patients with type 2 diabetes. The discrepancy between our study and that of Nielsen and colleagues may be related to species and/or condition. However, it should be noted that subsarcolemmal lipids also did not correlate with insulin sensitivity following aerobic training in the same report by Nielsen et al. [5], with five participants who displayed pronounced 
reductions in subsarcolemmal lipids having only modest changes in insulin sensitivity and one participant who had increased subsarcolemmal lipids with modestly improved insulin sensitivity [5]. Therefore, based on our observations, it appears that lipid accumulation in the subsarcolemmal region is not a direct cause and/or requirement for the development of insulin resistance in muscle of obese rodents. Presumably, and similarly to biochemical determinations of intramuscular lipids, lipid droplet accumulation in the subsarcolemmal region probably reflects alterations in cellular lipid homeostasis, rather than being a direct cause of insulin resistance.

Mitochondrial subcellular alterations and fatty acid metabolism

A decrease in mitochondrial fatty acid oxidation has been proposed to contribute to lipid accumulation $[8,36]$. Therefore regional differences in mitochondrial content and/or function may help to explain subcellular differences in lipid droplets. In the current study, muscle mDNA was increased in muscles of obese animals, which probably accounted for the increases in subsarcolemmal and intermyofibrillar mitochondrial density. Previously, moreover, we had shown that fatty acid oxidation in isolated subsarcolemmal and intermyofibrillar mitochondria remained unaltered (intermyofibrillar) or was increased (subsarcolemmal) [9] in the same animal model. The increase in subsarcolemmal mitochondrial density and fatty acid oxidation may represent compensatory mechanisms designed to prevent lipid droplet accumulation in the subsarcolemmal region. Therefore, reduced mitochondrial fatty acid oxidation apparently cannot account for the observed intramuscular lipid accumulation, a notion that is consistent with contemporary mitochondrial literature [37-41].

However, this literature is based on in vitro measurements that are optimised to determine the capacity of mitochondrial function and therefore may not represent the in vivo oxidative flux. In support of this, in the obese Zucker rat, despite increased mitochondrial density as shown here and elsewhere $[9,38]$, and despite increased in vitro mitochondrial function [9], we (present study) and others [41] found that rates of total muscle fatty acid oxidation were decreased, while, as found here, rates of triacylglycerol esterification were increased. It should be noted that the notion that fatty acid oxidation is reduced in obese Zucker rats $[15,42,43]$ is controversial. Nevertheless, the discrepancy between mitochondrial oxidative capacity and in vivo oxidative flux is echoed in the Zucker diabetic fatty rat, a model of type 2 diabetes where mitochondrial content and isolated mitochondrial function are increased [14], yet in vivo oxidative capacity, as assessed by ${ }^{31} \mathrm{P}$ magnetic resonance spectroscopy, is unaltered [44]. Several mechanisms may explain these data, including the observation that levels of malonyl-CoA, a biological inhibitor of carnitine palmitoyl-transferase I and mitochondrial fatty acid oxidation, are increased in obesity and in individuals with type 2 diabetes [45]. Combined, these data suggest the possibility that fewer fatty acids are transported into mitochondria in obese insulin-resistant muscle, a phenomenon that could contribute to lipid accumulation.

\section{Intramyocellular fatty acid trafficking}

To ascertain whether lipids are trafficked away from mitochondria in the obese animal, we incubated muscle in the presence of $\left[{ }^{3} \mathrm{H}\right]$ palmitate and subsequently isolated subsarcolemmal and intermyofibrillar mitochondria. The tibialis anterior muscle is not appropriate for this in vitro analysis, and so we used the soleus muscle, another oxidative muscle that also displays insulin resistance and lipid accumulation in these animals [46], for these studies. The results with this approach suggest that fewer lipids are transported into intermyofibrillar mitochondria in the in vivo situation. This finding is somewhat surprising, given the now wellrecognised observation that rates of fatty acid transport in the obese Zucker rat are substantially increased [9, 15, 41]. Nevertheless, it appears that less $\left[{ }^{3} \mathrm{H}\right]$ palmitate was transported into intermyofibrillar mitochondria, despite a greater delivery of fatty acids into the muscle's interior, suggesting that lipids were trafficked away from the mitochondria. The observed phenomenon was unlikely to have been caused by decreased transport capacity into mitochondria, as we have previously shown that carnitine palmitoyl-transferase I activity and rates of mitochondrial fatty acid oxidation are not reduced in these mitochondria [9]. Combined, these data suggest that lipids were trafficked away from the intermyofibrillar mitochondria in an unknown manner.

Given the proximity of intermyofibrillar mitochondria to the majority of lipid droplets in the obese animals, we hypothesised that triacylglycerol esterification enzymes would be increased in the intermyofibrillar region, providing a mechanism for the apparent stealing of lipids away from mitochondrial oxidation. However, similarly to previous reports [47, 48], increases in whole-muscle content of several esterification enzymes were not observed. In addition, lipin-1 and DGAT1 were not detectable on intermyofibrillar mitochondria, while GPAT1 content was unaltered with obesity. We cannot rule out the possibility that levels of these enzymes were increased specifically on the endoplasmic reticulum in the intermyofibrillar region. In addition, adipose tissue triacylglycerol lipase protein was unaltered (lean $100 \pm 4$, obese $89 \pm 4$ arbitrary units), while hormone-sensitive lipase cellular protein was increased (lean $100 \pm 6$, obese $138 \pm 16$ arbitrary units), suggesting 
that decreased lipolysis cannot account for the observed trafficking of lipids into lipid droplets. Alternatively, the observation that fewer lipids are transferred into intermyofibrillar mitochondria may simply reflect a situation where mitochondria are already oversupplied with lipids [49]. Nevertheless, as far as we can determine, ours is the first attempt to directly show that fatty acid transport into mitochondria is decreased in insulin-resistant muscle, possibly providing a potential mechanism for lipid accumulation in the intermyofibrillar region.

\section{Summary}

In the current study, we determined the subcellular distribution of lipids in insulin-resistant skeletal muscle. While others have suggested that subsarcolemmal lipids contribute to attenuations in insulin signalling [5], the current data suggest that subsarcolemmal lipids are not required for the development of insulin resistance, as insulin-resistant white muscles were devoid of lipids in the subsarcolemmal region. Therefore, similarly to increases in total triacylglycerol content, lipid droplet accumulation in the subsarcolemmal region is likely to simply reflect alterations in total cellular lipid homeostasis. In addition, we provide evidence that in insulin-resistant muscle fatty acids are trafficked away from intermyofibrillar mitochondria and probably into lipid droplets in the intermyofibrillar region. While the underlying mechanism for this diversion of fatty acids remains unknown, we have provided evidence that this is not a direct effect of increased triacylglycerol esterification enzyme content on intermyofibrillar mitochondria.

Acknowledgements This work was funded by the Natural Sciences and Engineering Research Council of Canada (NSERC; to G.P. Holloway and A. Bonen). A. Bonen is the Canada Research Chair in Metabolism and Health. Equipment was purchased with assistance from NSERC (to A. Bonen) and the Canada Foundation for Innovation (to G.P. Holloway).

Contribution statement JL performed experiments, interpreted data and wrote the paper. LS, XXH and AC performed experiments, interpreted data and edited the manuscript. $\mathrm{AB}$ designed experiments, interpreted data and edited the manuscript. GPH designed and performed experiments, interpreted data and edited the manuscript. All authors have approved the final version of the manuscript.

Duality of interest The authors declare that there is no duality of interest associated with this manuscript.

\section{References}

1. Muoio DM (2010) Intramuscular triacylglycerol and insulin resistance: guilty as charged or wrongly accused? Biochim Biophys Acta 1801:281-288
2. Chavez JA, Summers SA (2010) Lipid oversupply, selective insulin resistance, and lipotoxicity: molecular mechanisms. Biochim Biophys Acta 1801:252-265

3. Palmer JW, Tandler B, Hoppel CL (1986) Heterogeneous response of subsarcolemmal heart mitochondria to calcium. Am J Physiol 250:H741-H748

4. Hoppeler H, Howald H, Conley K et al (1985) Endurance training in humans: aerobic capacity and structure of skeletal muscle. J Appl Physiol 59:320-327

5. Nielsen J, Mogensen M, Vind BF et al (2009) Increased subsarcolemmal lipids in type 2 diabetes. Effect of training on localization of lipids, mitochondria and glycogen in sedentary human skeletal muscle. Am J Physiol Endocrinol Metab 298: E708-E713

6. Palmer JW, Tandler B, Hoppel CL (1977) Biochemical properties of subsarcolemmal and interfibrillar mitochondria isolated from rat cardiac muscle. J Biol Chem 252:8731-8739

7. Hoppel C, Kerner J, Turkaly P, Minkler P, Tandler B (2002) Isolation of hepatic mitochondrial contact sites: previously unrecognized inner membrane components. Anal Biochem 302:60-69

8. Kelley DE, He J, Menshikova EV, Ritov VB (2002) Dysfunction of mitochondria in human skeletal muscle in type 2 diabetes. Diabetes 51:2944-2950

9. Holloway GP, Benton CR, Mullen KL et al (2009) In obese rat muscle transport of palmitate is increased and is channeled to triacylglycerol storage despite an increase in mitochondrial palmitate oxidation. Am J Physiol Endocrinol Metab 296:E738E747

10. Cogswell AM, Stevens RJ, Hood DA (1993) Properties of skeletal muscle mitochondria isolated from subsarcolemmal and intermyofibrillar regions. Am J Physiol 264:C383-C389

11. Krieger DA, Tate CA, McMillin-Wood J, Booth FW (1980) Populations of rat skeletal muscle mitochondria after exercise and immobilization. J Appl Physiol 48:23-28

12. Benton CR, Nickerson JG, Lally J et al (2008) Modest PGClalpha overexpression in muscle in vivo is sufficient to increase insulin sensitivity and palmitate oxidation in subsarcolemmal, not intermyofibrillar, mitochondria. J Biol Chem 283:4228-4240

13. Benton CR, Holloway GP, Campbell SE et al (2008) Rosiglitazone increases fatty acid oxidation and fatty acid translocase (FAT/CD36) but not carnitine palmitoyltransferase I in rat muscle mitochondria. J Physiol 586:1755-1766

14. Holloway GP, Gurd BJ, Snook LA, Lally J, Bonen A (2010) Compensatory increases in nuclear $\mathrm{PGC} 1 \alpha$ protein are primarily associated with subsarcolemmal mitochondrial adaptations in ZDF rats. Diabetes 59:819-828

15. Luiken JJ, Arumugam Y, Dyck DJ et al (2001) Increased rates of fatty acid uptake and plasmalemmal fatty acid transporters in obese Zucker rats. J Biol Chem 276:40567-40573

16. Holloway GP, Snook LA, Harris RJ, Glatz JF, Luiken JJ, Bonen A (2011) In obese Zucker rats, lipids accumulate in the heart despite normal mitochondrial content, morphology and long-chain fatty acid oxidation. J Physiol 589:169-180

17. Wendel AA, Lewin TM, Coleman RA (2009) Glycerol-3phosphate acyltransferases: rate limiting enzymes of triacylglycerol biosynthesis. Biochim Biophys Acta 1791:501-506

18. Vock R, Hoppeler H, Claassen H et al (1996) Design of the oxygen and substrate pathways. VI. Structural basis of intracellular substrate supply to mitochondria in muscle cells. J Exp Biol 199:1689-1697

19. Shaw CS, Jones DA, Wagenmakers AJM (2008) Network distribution of mitochondria and lipid droplets in human muscle fibres. Histochem Cell Biol 129:65-72

20. Benton CR, Holloway GP, Han X-X et al (2010) Increased levels of peroxisome proliferator-activated receptor gamma, coactivator 
1 alpha (PGC-1alpha) improve lipid utilisation, insulin signalling and glucose transport in skeletal muscle of lean and insulinresistant obese Zucker rats. Diabetologia 53:2008-2019

21. Alkhateeb H, Chabowski A, Glatz JFC, Luiken JFP, Bonen A (2007) Two phases of palmitate-induced insulin resistance in skeletal muscle: impaired GLUT4 translocation is followed by a reduced GLUT4 intrinsic activity. Am J Physiol Endocrinol Metab 293:E783-E793

22. Dyck DJ, Peters SJ, Glatz J et al (1997) Functional differences in lipid metabolism in resting skeletal muscle of various fiber types. Am J Physiol 272:E340-E351

23. Holloway GP, Thrush AB, Heigenhauser GJF et al (2007) Skeletal muscle mitochondrial FAT/CD36 content and palmitate oxidation are not decreased in obese women. Am J Physiol Endocrinol Metab 292:E1782-E1789

24. Holloway GP, Bezaire V, Heigenhauser GJF et al (2006) Mitochondrial long chain fatty acid oxidation, fatty acid translocase/CD36 content and carnitine palmitoyltransferase I activity in human skeletal muscle during aerobic exercise. J Physiol 571:201-210

25. Campbell SE, Tandon NN, Woldegiorgis G, Luiken JJFP, Glatz JFC, Bonen A (2004) A novel function for fatty acid translocase (FAT)/CD36: involvement in long chain fatty acid transfer into the mitochondria. J Biol Chem 279:36235-36241

26. Crane JD, Devries MC, Safdar A, Hamadeh MJ, Tarnopolsky MA (2010) The effect of aging on human skeletal muscle mitochondrial and intramyocellular lipid ultrastructure. J Gerontol A Biol Sci Med Sci 65:119-128

27. Thiebaud D, Jacot E, DeFronzo RA, Maeder E, Jequier E, Felber JP (1982) The effect of graded doses of insulin on total glucose uptake, glucose oxidation, and glucose storage in man. Diabetes 31:957-963

28. Phillips SM, Green HJ, Tarnopolsky MA, Heigenhauser GF, Hill RE, Grant SM (1996) Effects of training duration on substrate turnover and oxidation during exercise. J Appl Physiol 81:21822191

29. Krssak M, Falk Petersen K, Dresner A et al (1999) Intramyocellular lipid concentrations are correlated with insulin sensitivity in humans: a ${ }^{1} \mathrm{H}$ NMR spectroscopy study. Diabetologia 42:113-116

30. Pan DA, Lillioja S, Kriketos AD et al (1997) Skeletal muscle triglyceride levels are inversely related to insulin action. Diabetes 46:983-988

31. Timmers S, Schrauwen P, de Vogel J (2008) Muscular diacylglycerol metabolism and insulin resistance. Physiol Behav 94:242251

32. Summers SA (2006) Ceramides in insulin resistance and lipotoxicity. Prog Lipid Res 45:42-72

33. Liu L, Shi X, Bharadwaj KG et al (2009) DGAT1 expression increases heart triglyceride content but ameliorates lipotoxicity. J Biol Chem 284:36312-36323

34. Goodpaster BH, He J, Watkins S, Kelley DE (2001) Skeletal muscle lipid content and insulin resistance: evidence for a paradox in endurance-trained athletes. J Clin Endocrinol Metabol $86: 5755-5761$
35. Dube JJ, Amati F, Stefanovic-Racic M, Toledo FG, Sauers SE, Goodpaster BH (2008) Exercise-induced alterations in intramyocellular lipids and insulin resistance: the athlete's paradox revisited. Am J Physiol Endocrinol Metab 294:E882-E888

36. Ritov VB, Menshikova EV, He J, Ferrell RE, Goodpaster BH, Kelley DE (2005) Deficiency of subsarcolemmal mitochondria in obesity and type 2 diabetes. Diabetes 54:8-14

37. Hancock CR, Han D-H, Chen M et al (2008) High-fat diets cause insulin resistance despite an increase in muscle mitochondria. Proc Natl Acad Sci USA 105:7815-7820

38. Turner N, Bruce CR, Beale SM et al (2007) Excess lipid availability increases mitochondrial fatty acid oxidative capacity in muscle: evidence against a role for reduced fatty acid oxidation in lipid-induced insulin resistance in rodents. Diabetes 56:20852092

39. Anderson E, Lustig M, Boyle $\mathrm{K}$ et al (2009) Mitochondrial $\mathrm{H}_{2} \mathrm{O}_{2}$ emission and cellular redox state link excess fat intake to insulin resistance in both rodents and humans. J Clin Invest 119:573-581

40. Mogensen M, Sahlin K, Fernström M et al (2007) Mitochondrial respiration is decreased in skeletal muscle of patients with type 2 diabetes. Diabetes 56:1592-1599

41. Han X-X, Chabowski A, Tandon NN et al (2007) Metabolic challenges reveal impaired fatty acid metabolism and translocation of FAT/CD36 but not FABPpm in obese Zucker rat muscle. Am J Physiol Endocrinol Metab 293:E566-E575

42. Crettaz M, Prentki M, Zaninetti D, Jeanrenaud B (1980) Insulin resistance in soleus muscle from obese Zucker rats. Involvement of several defective sites. Biochem J 186:525-534

43. Penicaud L, Ferre P, Terretaz J et al (1987) Development of obesity in Zucker rats. Early insulin resistance in muscles but normal sensitivity in white adipose tissue. Diabetes 36:626-631

44. De Feyter HM, Lenaers E, Houten SM et al (2008) Increased intramyocellular lipid content but normal skeletal muscle mitochondrial oxidative capacity throughout the pathogenesis of type 2 diabetes. FASEB J 22:3947-3955

45. Bandyopadhyay GK, Yu JG, Ofrecio J, Olefsky JM (2006) Increased malonyl-CoA levels in muscle from obese and type 2 diabetic subjects lead to decreased fatty acid oxidation and increased lipogenesis; thiazolidinedione treatment reverses these defects. Diabetes 55:2277-2285

46. Franch J, Knudsen J, Ellis BA, Pedersen PK, Cooney GJ, Jensen J (2002) Acyl-CoA binding protein expression is fiber type-specific and elevated in muscles from the obese insulin-resistant Zucker rat. Diabetes 51:449-454

47. Thrush AB, Brindley DN, Chabowski A, Heigenhauser GJ, Dyck DJ (2009) Skeletal muscle lipogenic protein expression is not different between lean and obese individuals: a potential factor in ceramide accumulation. J Clin Endocrinol Metab 94:5053-5061

48. Li M, Paran C, Wolins NE, Horowitz JF (2011) High muscle lipid content in obesity is not due to enhanced activation of key triglyceride esterification enzymes or to the suppression of lipolytic proteins. Am J Physiol Endocrinol Metab 300:E699-E707

49. Koves TR, Ussher JR, Noland RC et al (2008) Mitochondrial overload and incomplete fatty acid oxidation contribute to skeletal muscle insulin resistance. Cell Metab 7:45-56 